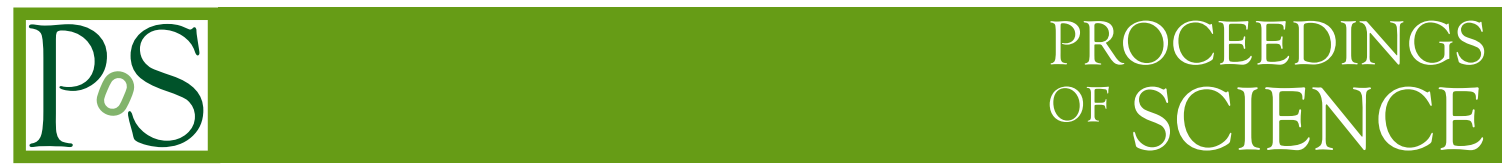

\title{
Searches for Axion Like Particles at the LHC
}

\author{
Kristof Schmieden ${ }^{a, *}$ on behalf of the ATLAS, CMS and TOTEM Collaborations \\ a Johannes Gutenberg Universität, \\ Mainz, Germany \\ E-mail: kristof.schmieden@cern.ch
}

Searching for axion-like particles is a very active field, as they are a potential candidate for dark matter. The LHC proves to be a versatile tool to search for axion-like particles in a wide mass range using a variety of production and decay modes, which are exploited using modern analysis techniques. An overview of the employed analysis strategies is presented together with a selected set of analyses from the LHC experiments covering the mass range from $0.5 \mathrm{GeV}$ to $600 \mathrm{GeV}$.

The Ninth Annual Conference on Large Hadron Collider Physics - LHCP2021

7-12 June 2021

Online

${ }^{*}$ Speaker 


\section{Introduction}

Axions were postulated by Pecci and Quinn [1] in 1977 as a solution to the so called strong $C P$ problem $[2,3]$ of quantum chromo dynamics. They obey a well defined relation between their mass and coupling to SM particles. Dropping the mass - coupling relation, any scalar or pseudoscalar particle with similar couplings to SM particles may be referred to as axion-like particle (ALP). While axions are generally expected to be very light in the sub-eV regime, ALPs can be arbitrarily heavy. At the LHC, ALP masses from $\mathrm{MeV}$ to hundreds of $\mathrm{GeV}$ are accessible via various production and decay modes. ALPs will always couple to photons and hence can be produced in photon-photon collisions at the LHC, which is a very clean signature when no pileup is present. They also may couple to gluons, $\mathrm{Z}$ bosons and Higgs bosons as well as leptons and quarks. Besides the photon fusion process the gluon-gluon fusion process is of interest at the LHC due to its large cross section. Furthermore the associated production with a $\mathrm{Z}$ boson is experimentally interesting as it allows for easy triggering. Also the production via the decay of a Higgs into a pair of ALPs or an ALP and a $\mathrm{Z}$ boson are of particular interest in the light of studying the potential Higgs-ALP coupling. ALPs may decay into a variety of particles. At the LHC final states with photons, muons, tauons and b-quarks as well as invisible decays are currently studied. The decay into photons is experimentally clean and of particular interest in combination with the production via photon-photon fusion. The decay into muons allows a precise mass reconstruction albeit having a smaller expected branching ratio compared to the decay into heavier particles like b-quarks and tauons. Searching for invisible ALPs is of interest as it also includes long lived particles escaping the detector.

In the following some selected analysis results are summarised. For a full list of the most recent results please see the web-pages of the LHC experiments [4, 5].

\section{ALPs in photon photon collisions}

At the LHC the search for ALPs in photon collisions is of particular interest due to its uniquely clean signature of the $\gamma \gamma \rightarrow a \rightarrow \gamma \gamma$ process, which would show up as a peak in the invariant di-photon mass spectrum over the continuous standard-model light-by-light scattering background. These analyses use data from ultra-peripheral heavy ion collisions (UPC). The relativistic nuclei of the heavy ion beams are an intense source of quasi-real photons. As the photon flux scales with the charge of the beam particles to the power of four, $\mathrm{Pb}$ ion beams produce a larger photon flux compared to the proton beams, despite their lower instantaneous luminosity. The low luminosity of $\mathrm{PbPb}$ collisions lead to pileup-free and therefore very clean UPC events. The maximum photon energy is given by the Lorentz factor of the beam particles divided by their radius, amounting to approximately $80 \mathrm{GeV}$ at LHC conditions. Using HI beams to study light-by-light scattering at the LHC was first proposed in Ref. [6]. Candidate events are selected by requiring exactly two photons above a low energy threshold in the detector and vetoing any additional activity. The photons are required to be back-to-back in the axial plane of the detector to suppress background from gluon-gluon fusion events as well as from electron-positron pairs misidentified as photons. Both, ATLAS [7] and CMS [8] have measured this process and calculated limits on the coupling of ALPs to photons $[9,10]$, which are the most stringent limits in the mass range from $5 \mathrm{GeV}$ to $30 \mathrm{GeV}$ to date. 
The light-by-light scattering process can also be measured in proton-proton collisions despite the large pile-up if the scattered protons are tagged. This analysis was performed by CMS and Totem [11] for invariant masses from $400 \mathrm{GeV}$ to $1900 \mathrm{GeV}$ but is not yet sensitive to the SM light-by-light scattering processes. $\mathrm{LHCb}$ is able to reconstruct di-photon events in proton proton collisions as is shown in Ref. [12], where $80 \mathrm{pb}^{-1}$ of publicly available LHCb data were analysed to set limits on the ALP - photon coupling in the mass range from $5 \mathrm{GeV}$ to $6.5 \mathrm{GeV}$. Derived prospects indicate that the range from $2 \mathrm{GeV}$ to $20 \mathrm{GeV}$ is accessible in LHC Runs 3 and 4.

\section{Higgs Portal: $h \rightarrow a a$ and $h \rightarrow Z a$}

Another interesting strategy is to look for Higgs bosons decaying into ALPs. If the Higgs boson is produced in association with a leptonically decaying Z-boson the events are easy to trigger and discriminate from background, allowing to study ALPs decaying into b-quarks. B-quark final states are of particular interest as their branching ratio is expected to be large, given that the ALP is a pseudoscalar particle that couples to b-quarks via a Yukawa interaction. In Ref. [13] the $q \bar{q} \rightarrow Z h \rightarrow l l a a, a a \rightarrow 4 b$ channel was chosen to search for ALPs between $15 \mathrm{GeV}$ and $30 \mathrm{GeV}$ mass. At those masses both b-quarks from an ALP are reconstructed as a single jet object, which is identified by a custom tailored boosted decision tree to contain two b-quarks. The invariant di-b mass is estimated using multivariate techniques as well.

Alternatively, one can also look for Higgs bosons produced via gluon fusion, which yield a 65 times larger cross section compared to the $Z h$ associated production. To facilitate the triggering on those events at least one of the axions is required to decay into muons or leptonically decaying tauons. Muons in the final state allow for a ten times better resolution on the ALP mass compared to b-quark final states. However, b-quark and tau final states yield larger branching ratios. Various analyses are performed by ATLAS and CMS to study different combinations of final states. The $h \rightarrow 4 \tau$ process is studied in Ref. [14]. To achieve a good mass resolution on the ALP, one $\tau$ from an ALP decay is required to decay into a $\mu$, while the other is allowed to decay in any one charged particle. The ALP mass is reconstructed from the final state muon plus the charged track. In Ref. [15] the decay $h \rightarrow 2 \tau 2 \mu$ is studied. The ALP mass is reconstructed from the 2-muon final state with high precision. One tau must decay into a muon as well, while the other is allowed to decay hadronically. Complementary to the previous analyses, also the ALPs decay into b-quarks and muons can be studied in the process $h \rightarrow 2 b 2 \mu$. The largest background in this channel originates from Drell-Yan + jet processess well as top quark decays. ATLAS uses a kinematic fit to optimise the 4-object invariant mass and a multivariate methods exploiting the di-jet and di-muon kinematic in order to select signal candidate events [16]. CMS [17] follows a different approach to discriminate between signal and background like events. The relative mass difference of the b-pair and muon-pair, and hence the difference of both reconstructed ALP masses within an event, as well as the difference of the invariant mass of the $2 b 2 \mu$ final state in comparison to the Higgs mass are used. Interestingly, both analyses observe a slight excess at similar masses, as shown in Fig. 1. For ATLAS the local significance is $3.3 \sigma$ at $m_{a}=52 \mathrm{GeV}$, while for CMS Fig. 1 shows a fluctuation just above the expected $95 \% \mathrm{CL}$ limit at $m_{a} \approx 49 \mathrm{GeV}$. No combination of both results has been carried out so far and it is unclear if both fluctuations are compatible given the mass difference. 

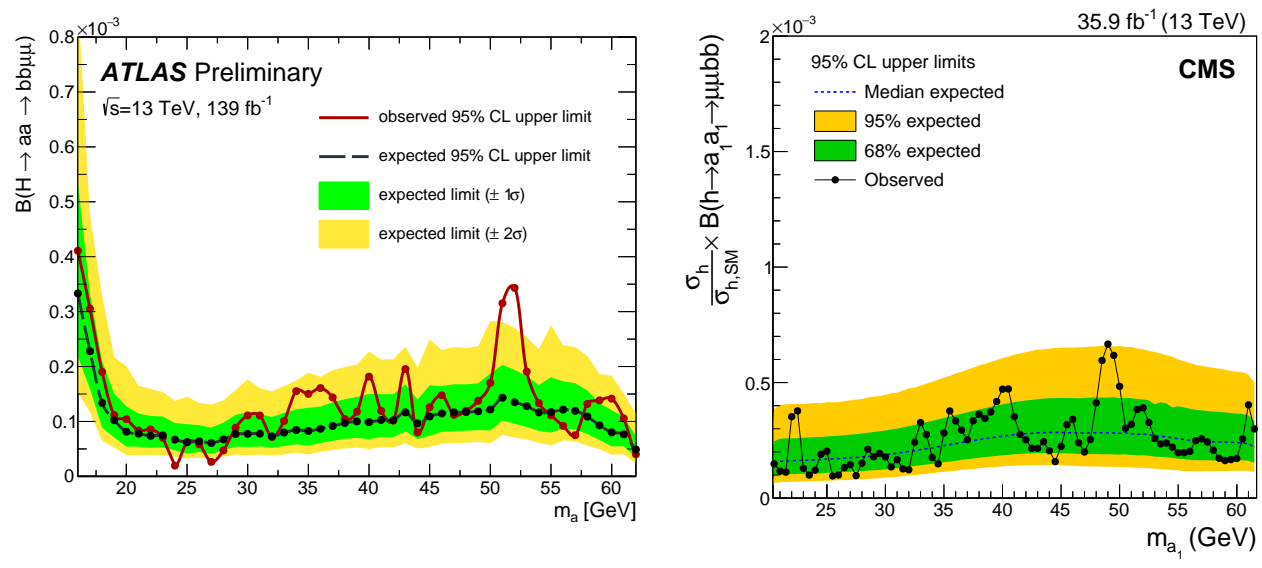

Figure 1: Observed and expected upper limits on the branching fraction $\mathrm{B}(h \rightarrow a a \rightarrow b b \mu \mu)$ at $95 \% \mathrm{CL}$ as a functino of the ALP mass $m_{a}$. Left the ATLAS result from [16], right the CMS result from [17].

Another approach is to look for Higgs boson decaying into one ALP and a $\mathrm{Z}$ boson. As the Z-boson is easily reconstructable, the ALP may decay into a difficult to reconstruct object, e.g. a pair of light quarks. This process was studied in Ref. [18] where the ALP is reconstructed as a single jet object. Neural networks relying on the track-based jet substructure information are used to discriminate between signal and background like events as well as to reconstruct the mass of the ALP in the range from $0.5 \mathrm{GeV}$ to $4 \mathrm{GeV}$. The largest background originates from $\mathrm{Z}$ bosons produced in association with one or more jets.

\section{Mono - X signatures}

Another class of analyses is looking for invisible decays of ALPs. This signature occurs if the ALP lives long enough to escape the detector, or if it decays into invisible particles. Hence the ALPs have to be produced in association with another, detectable, particle. As the detected particle is kinematically unbalanced in the event these signatures are commonly referred to as 'mono-X' signatures. Possible channels are $Z \rightarrow a \gamma$ [19], $h \rightarrow a Z$ [20] or $g g \rightarrow a g$ [21] which are all sensitive to different couplings of the ALP. In all cases the events exhibit significant missing transverse energies of typically $E_{\mathrm{T}}^{\text {miss }}>200 \mathrm{GeV}$. However, it is not possible to reconstruct the ALP masses. The results are interpreted either in terms of limits on effective field theory operators or in terms of specific models, e.g. a 2HDM plus ALP model as shown in [20]. None of the analyses observed a significant excess over the background expectation.

\section{Conclusions}

The LHC proves to be a versatile tool to search for axion-like particles in various production and decay channels. A a wide range of ALP masses from $0.5 \mathrm{GeV}$ to $600 \mathrm{GeV}$ has been studied in various analyses by ATLAS, CMS. While no significant excess of events has been observed over the standard model background, the $h \rightarrow a a \rightarrow 2 b 2 \mu$ channel shows an interesting fluctuation around a mass of $50 \mathrm{GeV}$ in both analyses from ATLAS [16] and CMS [17], certainly worth investigating further. 


\section{References}

[1] Peccei, R. D. and Quinn, Helen R., CP Conservation in the Presence of Pseudoparticles, Phys. Rev. Lett. 38, 1440. https://doi .org/10.1103/PhysRevLett. 38.1440

[2] Steven Weinberg, A New Light Boson?, Phys. Rev. Lett. 40, 223. https://doi.org/10. 1103/PhysRevLett. 40.223

[3] F. Wilczek, Problem of Strong $P$ and T Invariance in the Presence of Instantons, Phys. Rev. Lett. 40, 279. https://doi .org/10.1103/PhysRevLett. 40.279

[4] CMS Collaboration, CMS Public Results, URL: http://cms-results.web.cern.ch/ cms-results/public-results/publications/

[5] ATLAS Collaboration, ATLAS public results, URL: https://twiki.cern.ch/twiki/ bin/view/AtlasPublic

[6] Dvid d'Enterria and Gustavo G. da Silveira, Observing Light-by-Light Scattering at the Large Hadron Collider, Phys. Rev. Lett. 111, 080405. https://doi.org/10.1103/ PhysRevLett. 111.080405

[7] ATLAS Collaboration The ATLAS experiment at the CERN Large Hadron Collider 2008 JINST 3 S08003. https: //doi . org/10 . 1088/1748-0221/3/08/s08003

[8] CMS Collaboration The CMS experiment at the CERN LHC 2008 JINST 3 S08004. https: //doi.org/10.1088/1748-0221/3/08/s08004

[9] ATLAS Collaboration, Measurement of light-by-light scattering and search for axion-like particles with $2.2 \mathrm{nb}^{-1}$ of $\mathrm{Pb}+\mathrm{Pb}$ data with the ATLAS detector, J. High Energ. Phys. 2021, 243 (2021). https://doi .org/10.1007/JHEP03(2021) 243

[10] CMS Collaboration, Evidence for light-by-light scattering and searches for axion-like particles in ultraperipheral PbPb collisions at $\sqrt{N N}=5.02 \mathrm{TeV}$, Physics Letters B, Volume 797, (2019), 134826. https://doi.org/10.1016/j.physletb.2019.134826

[11] CMS and Totem Collaborations, First search for exclusive diphoton production at high mass with intact protons in proton-proton collisions at $\sqrt{s}=13 \mathrm{TeV}$ at the LHC, CMS-PAS-EXO18-014. https://cds. cern.ch/record/2725141

[12] X. Cid Vidal, A. Mariotti, D. Redigolo, F. Sala and K. Tobioka, New Axion Searches at Flavor Factories, JHEP 01 (2019), 113. https: / /doi .org/10 . 1007/JHEP01(2019) 113

[13] ATLAS Collaboration, Search for Higgs boson decays into two new low-mass spin-0 particles in the $4 b$ channel with the ATLAS detector using pp collisions at $\sqrt{s}=13 \mathrm{TeV}$, Phys. Rev. D 102 (2020) no.11, 112006. https: //doi .org/10.1103/PhysRevD. 102 . 112006

[14] CMS Collaboration, Search for light pseudoscalar boson pairs produced from decays of the $125 \mathrm{GeV}$ Higgs boson in final states with two muons and two nearby tracks in pp collisions at 
$\sqrt{s}=13$ TeV. Phys. Lett. B 800 (2020), 135087. https: //doi .org/10.1016/j . physletb. 2019.135087

[15] CMS Collaboration, Search for a light pseudoscalar Higgs boson in the boosted $\mu \mu \tau \tau$ final state in proton-proton collisions at $\sqrt{s}=13 \mathrm{TeV}$, Journal of High Energy Physics volume 2020, Article number: 139 (2020). https://doi .org/10 . 1007/JHEP08(2020) 139

[16] ATLAS Collaboration, Search for Higgs boson decays into a pair of pseudoscalar parti-

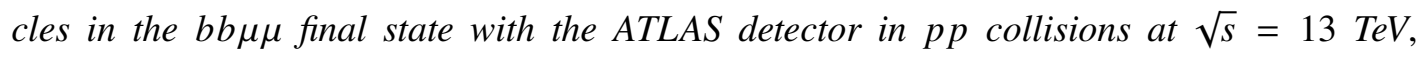
[arXiv:2110.00313 [hep-ex]]. https://arxiv.org/abs/2110.00313

[17] CMS Collaboration, Search for an exotic decay of the Higgs boson to a pair of light pseudoscalars in the final state with two muons and two b quarks in pp collisions at $13 \mathrm{TeV}$, Phys. Lett. B 795 (2019), 398-423. https: / /doi .org/10 . 1016/j . physletb. 2019.06. 021

[18] ATLAS Collaboration, The Search for Higgs Boson Decays into a Z Boson and a Light Hadronically Decaying Resonance Using $13 \mathrm{TeV}$ pp Collision Data from the ATLAS Detector, Phys. Rev. Lett. 125, 221802. https: //doi .org/10.1103/PhysRevLett.125. 221802

[19] ATLAS Collaboration, Search for dark matter in association with an energetic photon in pp collisions at $\sqrt{s}=13 \mathrm{TeV}$ with the ATLAS detector, Journal of High Energy Physics volume 2021, Article number: 226 (2021). https: //doi .org/10 . 1007/JHEP02(2021) 226

[20] CMS Collaboration, Search for dark matter produced in association with a leptonically decaying $Z$ boson in proton-proton collisions at $\sqrt{s}=13 \mathrm{TeV}$ The European Physical Journal C volume 81, Article number: 13 (2021). https://doi.org/10.1140/epjc/ s10052-021-08959-3

[21] ATLAS Collaboration, Search for new phenomena in events with an energetic jet and missing transverse momentum in p collisions at $\sqrt{s}=13 \mathrm{TeV}$ with the ATLAS detector, Phys. Rev. D 103 (2021) no.11, 112006. https://doi .org/10.1103/PhysRevD.103.112006 